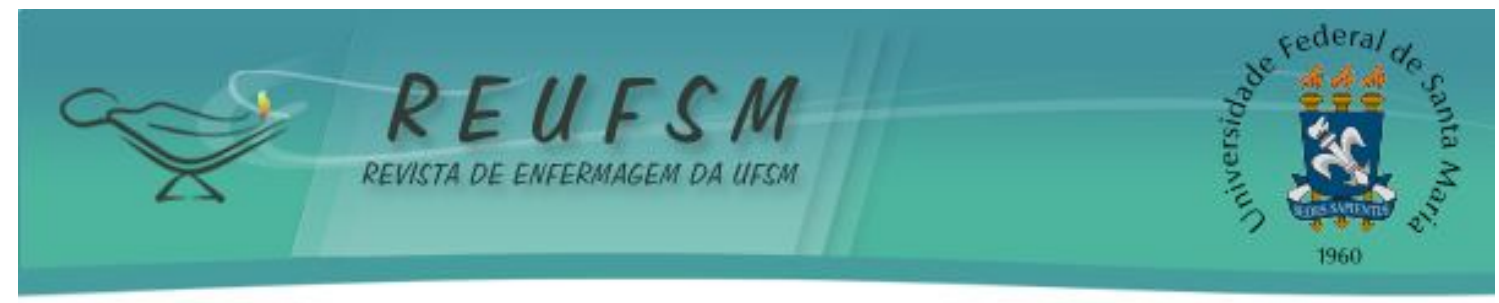

ARTIGO ORIGINAL

\title{
A METODOLOGIA DE CALLISTA ROY APLICADA EM CLIENTES SUBMETIDOS À INTERVENÇAO HEMODINÂMICA
}

\author{
THE METHODOLOGY APPLIED CALLIST A ROY CUST OMERS SUBMITTED FOR \\ INTERVENTION HEMODY NAMICS
}

\section{LA METODOLOGÍA DE CALLIST A ROY APLICADA AL CLIENTE SOMETIDO A LA INTERVENCIÓN HEMODINÁMICA}

\author{
Ivete Maroso Krauzer $^{1}$ \\ Dania Brocardo ${ }^{2}$ \\ Telma Scarsi ${ }^{3}$
}

\begin{abstract}
RESUMO: Trata-se de uma pesquisa qualitativa, do tipo exploratório descritiva, que identificou o perfil de saúde dos clientes em tratamento hemodinâmico e desenvolveu 0 Processo de Enfermagem (PE), fundamentado em Callista Roy. Realizou-se com 52 clientes, no Centro de Hemodinâmica de um hospital privado, de fevereiro a maio de 2010. Utilizouse um questionário semiestruturado para coleta de dados. A maioria tinha mais de 60 anos, aposentados, casados, moradores de Chapecó, com ensino fundamental. Os fatores de risco principais foram: idade acima de 60 anos, a Hipertensão Arterial Sistêmica (HAS), obesidade e vida sedentária. Os principais diagnósticos de enfermagem foram: ansiedade relacionada à morte (100\%); risco de dor aguda (85\%) e integridade da pele prejudicada (75\%). Os resultados obtidos podem contribuir para a enfermagem embasar o cuidado no $\mathrm{PE}$, identificar os fatores de risco, atuar na prevenção e num serviço de alta complexidade que exige conhecimentos especializados.
\end{abstract}

Descritores: Cuidados de enfermagem; Hemodinâmica; Perfil de saúde.

ABSTRACT: This is a qualitative exploratory and descriptive study that identified the health profile of the clients in treatment hemodynamic and developed the Nursing Process (EP) based on Callista Roy. It was held with 52 clients in Central Homodynamic in a private hospital from February to May 2010. We used a questionnaire for semi-structured data collection. Most had more than 60 years, retired, married, living Chapecó with school. The major risk factors were age over 60 years, hypertension, obesity and sedentary lifestyle. The major nursing diagnoses were: anxiety related to death $(100 \%)$, risk of acute pain ( $85 \%$ ) and impaired skin integrity (75\%). The results can contribute to base the nursing care in the EP, to identify risk factors, prevention work and service of high complexity requiring specialized knowledge.

Descriptors: Nursing care; Hemodynamics; Health profile.

RESUMEN: Se trata de un estudio cualitativo, de tipo exploratorio-descriptivo, que identificó el perfil de salud de los pacientes en tratamiento hemodinámico y desarrolló el Proceso de Enfermería (PE) fundamentado en Callista Roy. Se llevó a cabo con 52 clientes en el Centro de Hemodinámica de un hospital privado, de febrero a mayo de 2010. Se utilizó un cuestionario para la recogida de datos semi-estructurados. La mayoría de los

\footnotetext{
1 Enfermeira. Mestre em Enfermagem. Docente da Universidade Comunitária da Região de Chapecó (UNOCHAPECÓ) e Universidade do Estado de Santa Catarina (UDESC). E-mail: ivetemaroso@hotmail.com ${ }^{2}$ Acadêmica de Enfermagem, 9a Fase. Universi dade Comunitária da Região de Chapecó (UNOCHAPECÓ). E-mail: dbrocardo@gmail.com

${ }^{3}$ Acadêmica de Enfermagem, 9ạ Fase. Universidade Comunitária da Região de Chapecó (UNOCHAPECÓ). E-mail: telmas@unochapeco.edu.br
} 


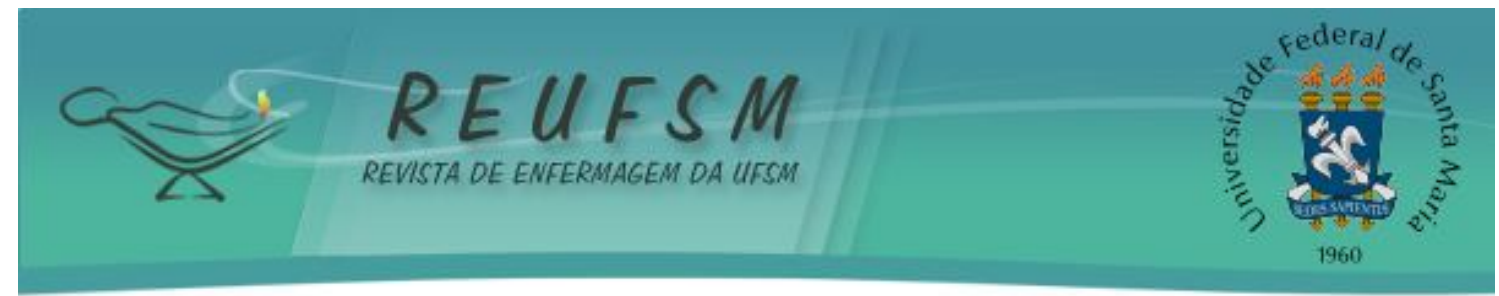

pacientes tenía más de 60 años, estaban jubilados, casados y vivían en la ciudad de Chapecó. Los principales factores de riesgo fueron: la edad superior a 60 años, la Hipertensión Arterial Sistêmica (HAS), la obesidad y el sedentarismo. Los diagnósticos principales fueron: la ansiedad relacionada con la muerte (el $100 \%)$, el riesgo de dolor agudo (el $85 \%$ ) y la integridad de la piel perjudicada (el 75\%). Los resultados pueden servir de base a los cuidados de enfermería en el PE, para identificar factores de riesgo, al trabajo de prevención y a los servicios de alta complejidad que requieren conocimientos especializados.

Descriptores: Atención de enfermería; Hemodinámica; Perfil de salud.

\section{INTRODUÇÃO}

A cardiologia intervencionista teve seu início no ano de 1905, quando Fritz Bleichroeder, ao dissecar as próprias veias, introduziu um cateter próximo ao coração, sem apresentar intercorrências. Em 1929, Waerner Forssman realizou o primeiro cateterismo cardíaco utilizando o método de fluoroscopia. ${ }^{1}$

No ano de 1966, o Instituto Dante Pazzanese de Cardiologia, em São Paulo, realizava a primeira cinecoronariografia. Em 1971, no Brasil e pela primeira vez no mundo, realizou-se uma recanalização mecânica de coronária. A partir da década de 70 houve uma verdadeira explosão de centros diagnósticos no Brasil, sendo marcado como a década de desenvol vimento desta área. ${ }^{2}$

A radiologia intervencionista comporta intervenções diagnósticas e/ ou terapêuticas guiadas por acesso percutâneo ou outro acesso, executadas geralmente sob anestesia local e/ ou sedação. ${ }^{3}$

0 aumento das doenças cardiovasculares contribuiu para o aprimoramento de estudos realizados em laboratórios de hemodinâmica, como: cateterismo cardíaco, angioplastia coronária com balão, com stent (endoprótese expansível); aterectomias (procedimento endovascular no qual a placa ateromatosa é removida); valvoplastias com balão (procedimento de escolha para o tratamento de pacientes com estenose das valvas cardíaca); atrioseptostomia (técnica em que se utiliza um pequeno cateter balão para promover a abertura do septo interatrial), dentre outros, o que possibilitou aprofundar os estudos de anatomia, fisiologia, etiologia e quadro clínico das doenças coronarianas. ${ }^{4}$

$\mathrm{Na}$ atualidade, estes procedimentos hemodinâmicos são plenamente justificáveis, tanto para os clientes quanto para o sistema de saúde, pois substituem as cirurgias complexas por procedimentos mais simples, diminuindo o risco, o tempo de internação e 0 custo total do procedimento. ${ }^{5}$

O serviço de hemodinâmica está em evolução contínua e a equipe multiprofissional deve estar em constante capacitação, de modo a poder prestar uma assistência qualificada. 0 enfermeiro hemodinamicista precisa desenvolver habilidades e competências para gerenciar um setor de alta complexidade, em que as responsabilidades são compartilhadas, mas dependem de um comando único.

0 autor refere que:

a responsabilidade em gerenciar a equipe, que muitas vezes não se limita somente à enfermagem, mas também a outros profissionais como equipes da radiologia, médica, secretárias, higiene e limpeza e da nutrição dietética. Outro grande desafio é como manter a equipe motivada e atualizada e ao mesmo tempo gerenciar os recursos materiais. ${ }^{1: 22}$ 


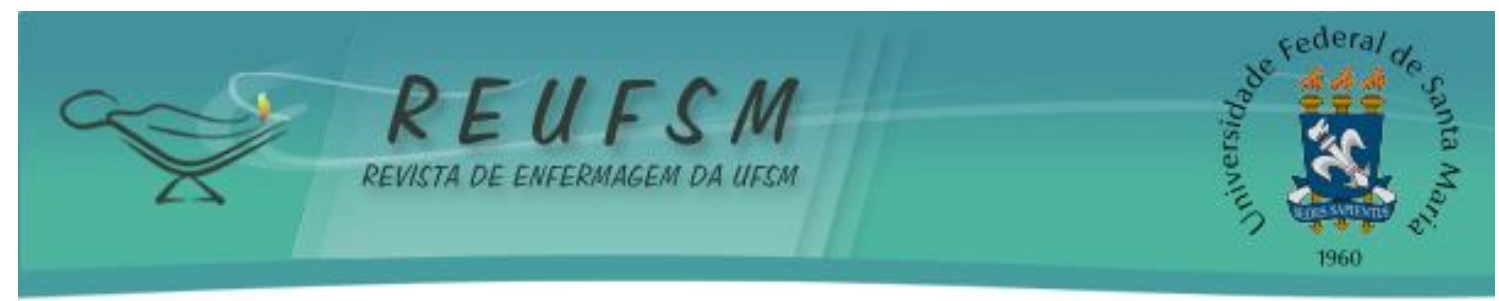

Além de gerenciar a equipe, o Enfermeiro necessita apoiar-se num modelo teórico para prestar assistência de qualidade. Trata-se de utilizar o Processo de Enfermagem (PE) como um instrumento metodológico que norteia as ações de enfermagem. Nesta pesquisa foram utilizados os pressupostos da teorista de Enfermagem Call ista Roy. ${ }^{6-7}$

A opção por abordar o cuidado sistematizado em clientes de um serviço de hemodinâmica ocorreu, levando em consideração os índices epidemiológicos alarmantes das doenças cardiovasculares, pois constituem a principal causa de mortalidade e morbidade, no Brasil e no mundo. ${ }^{8}$

A maioria das doenças cardiovasculares leva, invariavelmente, à necessidade de uma intervenção cirúrgica, em um serviço hospitalar especializado. A importância deste trabalho está aliada à implantação recente do Centro de Hemodinâmica e do Processo de Enfermagem no hospital em foco.

Diante do exposto, foi estabelecido como objetivo: identificar o perfil de saúde dos clientes assistidos no Centro de Hemodinâmica e aplicar o Processo de Enfermagem, de acordo com Callista Roy.

Roy estabelece cinco fases para realizar o Processo de Enfermagem: a investigação ou avaliação comportamental; os diagnósticos de Enfermagem; 0 estabelecimento de metas; plano para implementação ou intervenção e a avaliação.

Considera-se relevante a presença do enfermeiro em serviços hospitalares de alta complexidade, desenvolvendo o Processo de Enfermagem embasado em modelos teóricos, que enfoquem o bem estar do cliente e de seu familiar. Além disso, a aplicação da Sistematização da Assistência de Enfermagem (SAE) ou PE visa o atendimento da legislação da enfermagem brasileira. ${ }^{9}$

\section{MÉTODO}

Esta pesquisa caracterizou-se como exploratória descritiva, realizada na 9a Fase do Curso de Enfermagem, da Universidade Comunitária da Região de Chapecó (UNOCHAPECÓ). Foi desenvolvida nos meses de fevereiro a maio de 2010, correspondendo a 240 horas de atividades, junto aos clientes que tiveram intercorrências cardíacas e vasculares, e submeteram-se a procedimentos cirúrgicos no Centro de Hemodinâmica de um hospital privado do oeste de Santa Catarina. Este Centro faz parte de um hospital que disponibiliza 78 leitos de internação, dispõe de 208 médicos associados e cerca de 547 colaboradores.

As horas utilizadas para desenvolver a pesquisa foram fixadas pelo plano de ensino do Estágio Curricular Supervisionado II $\left(E C S\right.$ II) ${ }^{10}$ e distribuíram-se da seguinte maneira: 30 horas foram destinadas a um estudo piloto com cinco clientes, realizado no segundo semestre de 2009, com o objetivo de aplicar e validar um instrumento de coleta de dados, composto por um questionário semiestruturado. O projeto piloto foi importante para fazer os ajustes no questionário e garantir uma homogeneidade na abordagem aos clientes.

Às 210 horas restantes foram destinadas a coleta de dados, realizada pelas mesmas acadêmicas do projeto piloto, sem vínculo empregatício com o hospital, de segunda a sexta-feira. As entrevistas ocorreram em dois momentos: a primeira ocorreu no Centro de Hemodinâmica, no período pós-operatório imediato e a segunda entrevista ocorreu durante a visita domiciliar (VD). Esta visita era combinada, por telefone, para ser realizada uma semana após a alta do cliente.

Os critérios de inclusão na pesquisa eram de clientes que tivessem sido submetidos a intervenções cirúrgicas, de segunda a sexta-feira, no período matutino ou vespertino. Nestes turnos as acadêmicas estavam presentes no Centro de Hemodinâmica. Como critérios de exclusão foram estabelecidos os clientes atendidos durante o período noturno, nos finais de semana e feriados ou quando a internação coincidia com 0 


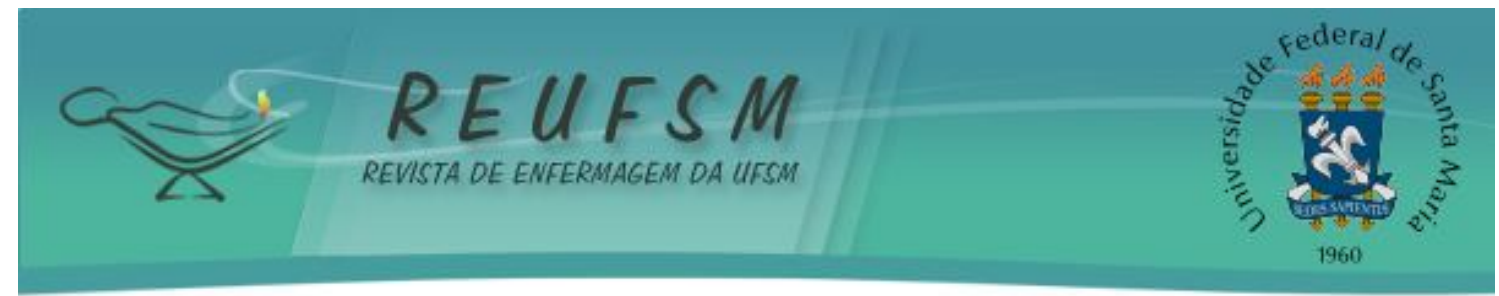

agendamento da VD. O modelo Roy oferece diretrizes para a enfermeira avaliar o nível de adaptação do indivíduo e compreende cinco fases, que caracterizam o Processo de Enfermagem, descritas a seguir. ${ }^{11}$

\section{Investigação ou Avaliação Comportamental}

A investigação comportamental é a fase em que são coletados os dados, ou seja, a realização da entrevista. ${ }^{7}$ É apresentada em dois tipos de avaliação: na primeira, a enfermeira realiza a coleta de respostas ou de comportamentos de saída da pessoa, em relação a cada um dos modos adaptativos. Na segunda, observa se existe uma resposta comportamental adaptativa ou ineficiente. Nesta avaliação, importam os dados referentes aos estímulos presentes, que podem afetar a adaptação da pessoa. ${ }^{7}$

\section{Diagnósticos de Enfermagem}

Roy propõe três métodos para realizar o diagnóstico de enfermagem, descritos a seguir: o primeiro é o diagnóstico por levantamento de problemas, onde há o uso de uma tipologia de diagnóstico (criada pela própria teorista) que relaciona os quatro modos adaptativos. ${ }^{11-12} \mathrm{O}$ segundo faz um diagnóstico relatando a resposta observada de modo conjunto com os estímulos mais influentes. No terceiro, ocorre um resumo das respostas em um ou mais modos adaptativos relacionados com o mesmo estímulo. ${ }^{11-12}$

\section{Estabelecimento de metas}

As metas são os comportamentos finais que a pessoa deve atingir. São registradas como comportamento do cliente indicativo de resolução do problema de adaptação. Inclui mudanças esperadas e a estrutura de tempo pode ser a curto ou longo prazo. Sempre que possível, as mudanças são estabel ecidas mutuamente com a pessoa, respeitando os direitos do indivíduo. ${ }^{4}$

\section{Planos para Implementação ou Intervenção}

As intervenções de enfermagem são planejadas com a finalidade de alterar ou controlar os estímulos focais ou contextuais. A implementação pode focar a ampliação da capacidade de enfrentamento da pessoa ou seu nível de adaptação, de forma que os estímulos totais permaneçam na capacidade de adaptação. Inclui a seleção e aplicação de atividades ou cuidados de enfermagem para promover a adaptação, modificando os estímulos ou fatores influentes no fortalecimento dos processos de enfrentamento. ${ }^{4}$

\section{Avaliação}

É a readaptação das metas e das intervenções, realizada com base nos dados de adaptação. Também denominada Evolução, é feito o julgamento da eficácia da intervenção de enfermagem, em relação ao comportamento do sistema humano. ${ }^{4}$

Estes diferentes modos adaptativos foram realizados durante as entrevistas com os clientes no Centro de Hemodinâmica e nas visitas domiciliares do pós-operatório. A abordagem aos clientes ocorreu de acordo com a Resolução 196/ 96 do Conselho Nacional de Saúde. 0 projeto foi submetido ao Comitê de Ética em Pesquisa da UNOCHAPECÓ e aprovado com registro n. 067/ 10. O Estágio foi supervisionado pela Enfermeira do Centro de Hemodinâmica e orientado por uma docente do Curso. 


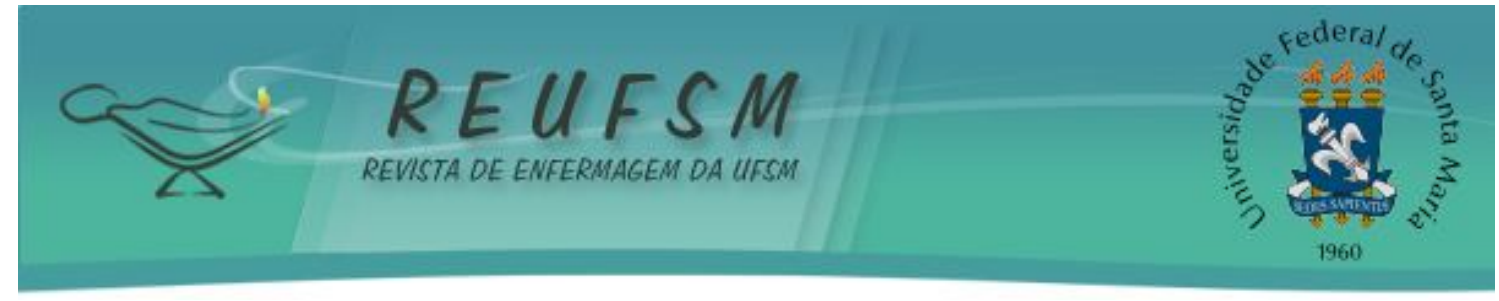

\section{RESULTADOS E DISCUSSÃO}

Os resultados obtidos foram descritos, no primeiro momento, com o perfil de saúde dos clientes atendidos, de modo a oferecer características sobre o tipo de clientela, por meio da identificação da faixa etária; do local de moradia; estado civil; da escolaridade; ocupação; dos fatores de risco para doenças cardiovasculares; Índice de Massa Corporal - IMC e dos tipos mais frequentes de intervenções cirúrgicas. No segundo momento foi delineado o Processo de Enfermagem.

\section{Perfil de saúde dos clientes assistidos}

A assistência foi realizada a 52 pessoas, contemplando 47,2\%do total dos clientes assistidos no período de fevereiro a maio de 2010, no Centro de Hemodinâmica. Destes, 24 clientes eram do sexo feminino $(46,1 \%)$ e 28 do sexo masculino $(53,85 \%$. Não houve óbitos neste período.

No período de março de 2009 a março de 2010 foram realizadas 1.232.506 internações no Brasil, por doenças cardiovasculares, das quais 32.529 pessoas foram a óbito (2,6\%). ${ }^{8}$ Em Santa Catarina das 51.769 internações registradas, 24.765 (47,8\%) eram clientes do sexo masculino e 27.003 (52.1\%) foram do sexo feminino. De mortalidade, encontrou-se 3.351 pessoas que foram a óbito $(6,4 \%)$ por doenças do aparelho circulatório, sendo 1.697 (50.6\%) foram do sexo masculino e 1654 (49.3\%) foram de pessoas do sexo feminino, neste mesmo período. ${ }^{8}$

Nota-se que o resultado encontrado no Centro de Hemodinâmica difere das estatísticas de Santa Catarina, pois no Estado aparecem mais pessoas do sexo feminino com morbidades do aparelho circulatório, entretanto morrem mais homens por estas causas. Cerca de $80,0 \%$ dos atendimentos ocorreu em clientes com mais de 60 anos, o que vem de encontro com a justificativa quanto à necessidade de avaliar periodicamente os idosos, no intuito de detectar problemas de saúde e fatores de risco relacionados com as alterações cardiovasculares. ${ }^{13}$

Do total de clientes, 31 (59,6\%) residiam no município de Chapecó, e os demais residiam na região oeste e extremo oeste de Santa Catarina. Sendo que este Centro atendia clientes conveniados e particulares.

Quando questionados sobre o estado civil, $36(69,2 \%)$ clientes se declararam casados; sete $(13,4 \%)$ viúvos; seis $(11,5 \%)$ divorciados e três $(5,7 \%)$ solteiros. No quesito escolaridade, $34(65,3 \%)$ clientes possuía ensino fundamental incompleto; dez (19,2\%) 0 ensino médio completo e oito (15,3\%) completaram o ensino superior. Quanto à ocupação, dez (19,2\%) pessoas atuavam como comerciantes; 12 (23,0\%) exerciam outra ocupação e 30 $(57,6 \%)$ deles estavam aposentados.

Entre os fatores de risco, destacaram-se: a idade (acima de 60 anos), os antecedentes familiares, a Hipertensão Arterial Sistêmica (HAS), obesidade, vida sedentária, alimentação rica em sódio e gordura, uso moderado de álcool, uso do tabaco, e o estresse.

Os principais fatores de risco para doenças cardiovasculares, identificados por outros estudos, incluem o tabagismo, a HAS e a hipercolesterolemia, seguidos por diabetes, obesidade e inatividade física. ${ }^{14}$ Nesta pesquisa, 31 clientes apresentaram hipertensão como patologia associada, que corresponde a $59,6 \%$ dos clientes assistidos.

Os clientes foram questionados sobre o consumo de cafeína e assim foram registradas $22(42,3 \%)$ pessoas que ingeriam café diariamente. Esse dado torna-se relevante à medida que 0 consumo exagerado gera um risco maior para alterações na pressão arterial. ${ }^{15}$ 


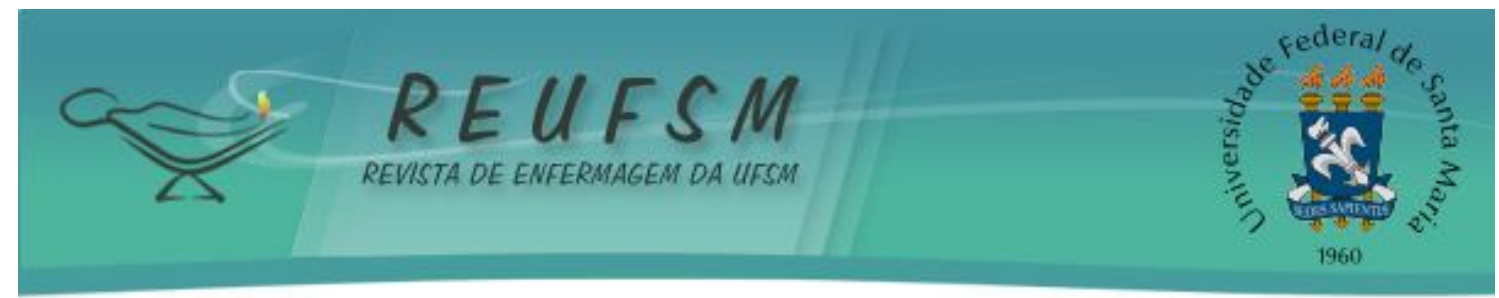

No registro do Índice de Massa Corporal (IMC) obtivemos o seguinte resultado: a maioria $(80,0 \%)$ apresentou alteração do IMC, entre 25 a $29.9 \mathrm{~kg} / \mathrm{m}^{2}$, com sobrepeso. Declararam ter uma vida sedentária e não costumavam cuidar de sua alimentação.

Em um estudo realizado na maior sala de emergência do Sul do Brasil a média de IMC foi de 28,1 , ou seja, $77 \%$ da amostra apresentou IMC maior que $25 \mathrm{~kg} / \mathrm{m} 2$, que indica sobrepeso. ${ }^{15}$ Nenhum cliente assistido nesta pesquisa apresentou obesidade de grau III (mórbida).

Em uma pesquisa realizada pelo Ministério da Saúde, que analisou a altura média dos brasileiros correlacionada ao IMC, nos períodos de 1974 a 1989 e de 1989 a 2002 notou-se que:

[...] os valores médios de IMC da população adulta masculina brasileira, confirmam a trajetória continuamente ascendente desse índice apenas nos homens. Entre mulheres a média do IMC aumentou significativamente somente no período de 1974 a 1989 e manteve-se estável no período de 1989 a 2002 . $^{8: 277}$

0 excesso de peso, com IMC maior de $25 \mathrm{~kg} / \mathrm{m} 2$, predispõe ao aparecimento de doenças cardiovasculares devido a problemas no metabolismo dos lipídeos, glicose e pressão arterial. Nesta condição ocorre menor utilização da glicose em resposta à ação da insulina nos tecidos periféricos acarretando um "menor consumo de glicose que faz com que seus níveis séricos tenham a tendência a se elevar, acarretando maior estímulo para a produção de insulina e consequente hiperinsulinemia". ${ }^{16: 4}$

As intervenções cirúrgicas mais frequentes foram o cateterismo cardíaco com a finalidade de realizar angioplastias e angiografias. Cateterismo cardíaco "é um procedimento diagnóstico invasivo em que cateteres arteriais e venosos radiopacos são introduzidos em vasos sanguíneos selecionados dos lados direito e esquerdo do coração". 17:538 Usualmente é feito com angiografia em que ocorre a injeção de contraste dentro do sistema vascular para obter uma imagem do coração e vasos. ${ }^{17}$ Angioplastia consiste da passagem de um fio guia na luz da artéria coronária por onde é injetado contraste radiográfico. Nesse guia são introduzidos balões infláveis, stents e cateteres de aterectomia com a finalidade de desobstruir o vaso e mantê-lo livre para a passagem do sangue. ${ }^{2}$

\section{Aplicação do modelo de Callista Roy}

A investigação comportamental foi avaliada por meio de respostas verbais e pela observação do cliente. As respostas comportamentais ineficientes foram descritas em clientes que manifestavam medo $(55,0 \%)$ em relação ao procedimento; insegurança em relação à equipe médica $(25,0 \%)$ principalmente em clientes que foram encaminhados de outros municípios e não conheciam a equipe que realizaria o procedimento. Incluíram-se nessa avaliação também os clientes que, apesar de alterações diagnosticadas, não tiveram mudanças em hábitos de vida que eram conhecidos como prejudiciais (20\%) como: tabagismo, alimentação inadequada, estresse, inatividade física.

Respostas comportamentais adaptativas foram identificadas em clientes que realizaram procedimentos diagnósticos e, após estes, iniciaram mudanças positivas em seu comportamento, que, aliado aos procedimentos terapêuticos, ajudaram na melhoria de sua qualidade de vida (restrição do consumo de álcool, diminuição da ingesta de sal e gordura animal, pesquisa de exercício físico).

O comportamento dos clientes relacionados aos seus modos de vida e a manifestação do nível adaptativo refletiu o uso dos mecanismos de enfrentamento. Por meio da observação do comportamento da pessoa, em relação aos modos adaptativos, a enfermeira pode identificar respostas adaptativas ou ineficientes em situações tanto de saúde ou de doença. ${ }^{18}$ 


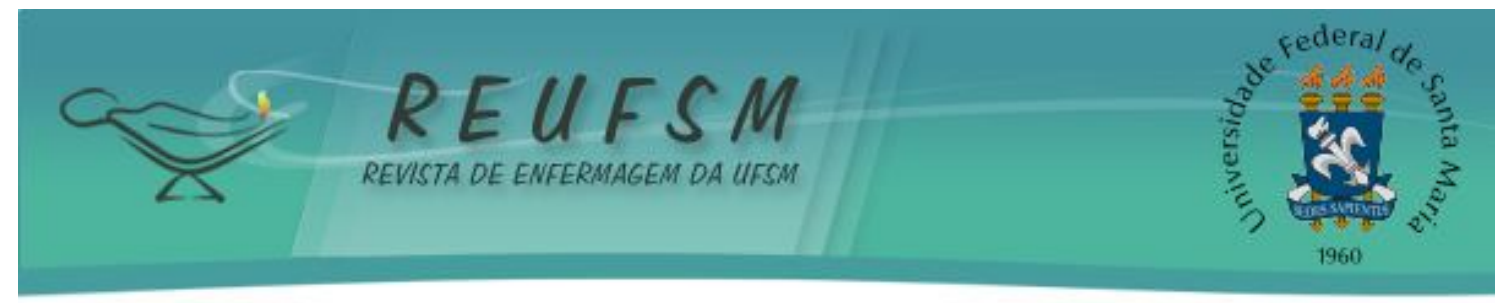

Os seis primeiros diagnósticos de enfermagem do cliente no pós-operatório, foram identificados por meio da tipologia da $\operatorname{NANDA}^{4}(2007-2008)^{19}$, pois, apesar de algumas diferenças entre a tipologia de Roy e a de NANDA, há uma forte relação entre as mesmas, o que pode ser fruto da participação de Callista Roy como membro da NANDA desde os primeiros trabalhos dessa taxonomia. ${ }^{20}$ Para identificar o sétimo diagnóstico, utilizamos 0 referencial de Carpenito ${ }^{5}$

Neste artigo optou-se por descrever os diagnósticos do período pós-operatório, haja vista que a maioria dos clientes não internava e chegavam ao Centro de Hemodinâmica em torno de meia hora antes do procedimento. A ação das acadêmicas ocorria na sala de recuperação pós-anestésica. No período trans-operatório não foi permitido o acompanhamento dos clientes pelas acadêmicas, devido ao espaço exíguo do Centro de Hemodinâmica.

Quadro 1. Diagnósticos de Enfermagem da NANDA Internacional (2007-2008) -Taxonomia I.

\begin{tabular}{|c|c|c|c|}
\hline $\begin{array}{lr}\text { DIAGNÓSTICOS } & \text { DE } \\
\text { ENFERMAGEM } & \text { NO } \\
\text { PERÍODO } & \text { PÓS- } \\
\text { OPERATÓRIO } & \end{array}$ & $\begin{array}{l}\text { PERCENTUAL } \\
\text { DE INCIDÊNCIA } \\
\text { NOS CLIENTES }\end{array}$ & CARACTERISTICAS DEFINIDORAS & $\begin{array}{l}\text { FATORES } \\
\text { RELACIONADOS }\end{array}$ \\
\hline $\begin{array}{l}\text { 1. Ansiedade } \\
\text { relacionada à morte }\end{array}$ & $100 \%$ & $\begin{array}{l}\text { Comportamentais: nervosismo; } \\
\text { Preocupações expressas em } \\
\text { razão de mudanças em eventos } \\
\text { da vida. } \\
\text { Simpáticas: aumento da pressão } \\
\text { sanguínea; boca seca. } \\
\text { Afetivas: aflito } \\
\text { Parassimpáticas: náuseas } \\
\text { Cognitivas: atenção prejudicada }\end{array}$ & $\begin{array}{l}\text { Ameaça de morte; } \\
\text { Ameaça ao estado de } \\
\text { saúde. }\end{array}$ \\
\hline 2. Risco de dor aguda & $85 \%$ & $\begin{array}{l}\text { Alterações na pressão } \\
\text { sanguínea. } \\
\text { Evidência observada de dor. }\end{array}$ & $\begin{array}{l}\text { Agentes lesivos } \\
\text { (introdução } \\
\text { cateteres) }\end{array}$ \\
\hline $\begin{array}{l}\text { 3. Integridade da pele } \\
\text { prejudicada }\end{array}$ & $75 \%$ & $\begin{array}{l}\text { Rompimento da superfície da } \\
\text { pele. }\end{array}$ & $\begin{array}{l}\text { Uso de cateter arterial } \\
\text { Uso de anticoagulante } \\
\mathrm{e} \quad \text { antiagregante } \\
\text { plaquetário prévio } \\
\text { durante } \\
\text { procedimento }\end{array}$ \\
\hline $\begin{array}{lr}\text { 4.Perfusão } & \text { tissular } \\
\text { ineficaz. } & \text { Tipo } \\
\text { cardiopulmonar } & \end{array}$ & $49 \%$ & $\begin{array}{l}\text { Alteração no } \quad \text { sistema de } \\
\text { condução cardíaca. Arritmias. }\end{array}$ & $\begin{array}{l}\text { Interrupção do fluxo } \\
\text { sanguíneo }\end{array}$ \\
\hline $\begin{array}{l}\text { 5. Perfusão tissular } \\
\text { ineficaz. Tipo renal }\end{array}$ & $42 \%$ & Oligúria e anúria. & $\begin{array}{l}\text { Problemas de troca } \\
\text { (Uso de contraste) }\end{array}$ \\
\hline 6. Risco de infecção & $24 \%$ & $\begin{array}{l}\text { Defesas primárias inadequadas } \\
\text { (pele rompida, tecido } \\
\text { traumatizado). } \\
\text { Procedimentos invasivos. }\end{array}$ & $\begin{array}{l}\text { Procedimento } \\
\text { invasivo. }\end{array}$ \\
\hline $\begin{array}{l}\text { 7. Risco de inibição da } \\
\text { cascata de coagulação }\end{array}$ & $16 \%$ & $\begin{array}{l}\text { Uso de medicamentos } \\
\text { intravenosos }\end{array}$ & $\begin{array}{l}\text { Procedimento } \\
\text { invasivo. }\end{array}$ \\
\hline
\end{tabular}

\footnotetext{
${ }^{4}$ North American Nursing Diagnosis Association

${ }^{5}$ Carpenito LJ . Diagnóstico de Enfermagem: aplicação à prática clínica 8a. ed. Porto Alegre: Artmed; 2002.
} 


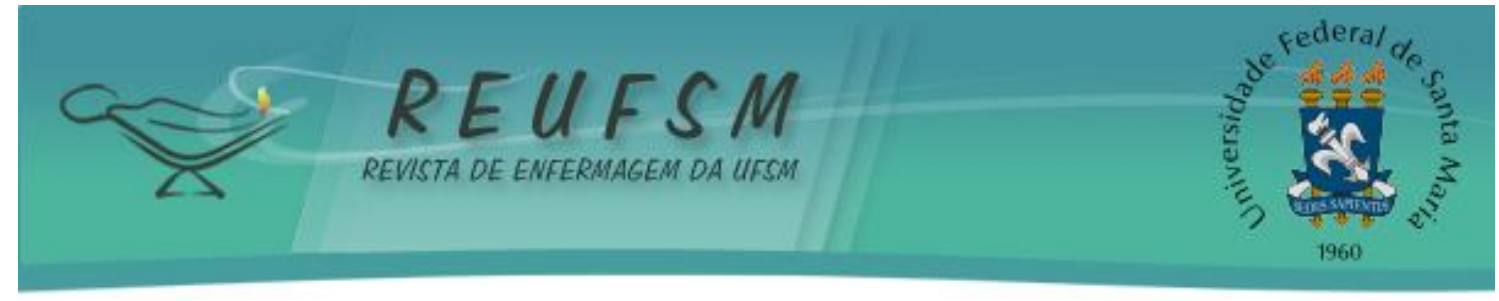

O estabelecimento de metas repousou sobre as possíveis reações tardias ao contraste injetado, monitoramento do volume urinário, além do estímulo a ingestão hídrica, visando à eliminação do contraste infundido. A atuação da enfermagem no pósprocedimento em cardiologia intervencionista, o foco de atenção das acadêmicas foi para as possíveis intercorrências no repouso do cliente.

O plano de Intervenção consistiu na elaboração das prescrições prioritárias, de modo a manter o membro puncionado em repouso, favorecendo a hemostasia da punção arterial, atentando para que fossem realizados movimentos suaves da extremidade do membro. Quando o cliente permanecia com compressão por peso, além de avaliação constante de possível presença de sangramento, o cliente era orientado a manter 0 posicionamento deste sobre o local da punção.

A fase de Avaliação, também chamada Evolução, foi realizada conforme 0 exemplo a seguir:

[09:40h Cliente A. Recebo cliente, feminina, 88 anos, casada, católica, aposentada, procedente da Colônia Cella, acompanhada pela filha ao Centro de Hemodinâmica para realizar angioplastia de Membro Inferior Direito, com os médicos H. e C. Apresenta-se ansiosa, comunicativa, deambulando com auxilio de bengala. Realizada entrevista de admissão, explicando como será o procedimento. Refere ter realizado um cateterismo diagnóstico há oito dias. Ao exame físico, realizada ausculta cardíaca com frequência e ritmo normal, na ausculta pulmonar murmúrios vesiculares bilaterais e na ausculta abdominal RHA (+) em todos os quadrantes. Apresenta úlcera varicosa na região tibial em Membro Inferior Esquerdo há nove anos. Encaminhada para a SRPA, puncionado AVP $\mathrm{n}$ - 20 em antebraço Esquerdo, mantido heparinizado, realizada tricotomia na região inguinal direita e em seguida foi encaminhada para procedimento. Às 11:20h, o cliente retorna para a SRPA, em pós angioplastia de Membro Inferior Esquerdo, com anestesia local. Apresenta-se calma, refere sentir-se bem, sinais vitais estáveis, mantém curativo oclusivo e compressivo em região inguinal $D$. Orientada a não forçar o membro puncionado e a qualquer desconforto comunicar a equipe. Às 12:30h foi liberada dieta branda, com boa aceitação. Cliente apresenta-se em processo adaptativo prejudicado e este se relaciona a ansiedade quanto ao procedimento (estímulo focal), e pela necessidade de internação (estímulo contextual). A presença da filha lhe garante segurança e inclui a família como fator predominante na sua recuperação.

Esta Evolução continuava durante a visita domiciliar realizada na primeira semana após a alta médica. Neste momento, eram avaliados os modos adaptativos desenvolvidos. Notou-se que $75 \%$ dos clientes tinham adaptação prejudicada, mas desenvolveram um comportamento de busca de saúde, relacionado às mudanças de hábitos pessoais; $15 \%$ desenvolveram um controle eficaz do regime terapêutico e 10\% apresentaram desobediência às orientações. 


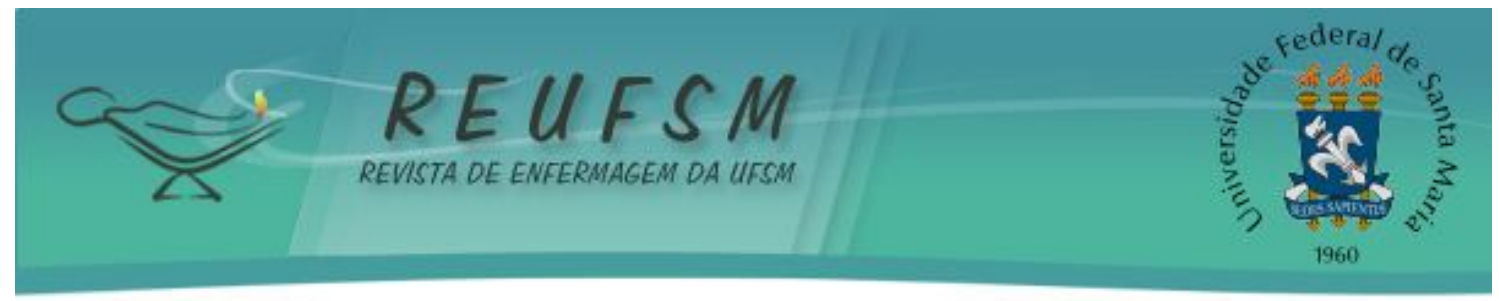

\section{CONSIDERAÇÕES FINAIS}

Quanto ao perfil dos clientes notou-se que a maioria tinha mais de 60 anos, eram aposentados, casados, moradores de Chapecó, com ensino fundamental. Os fatores de risco encontrados foram corroborados pela literatura como: a idade (acima de 60 anos), os antecedentes familiares, a Hipertensão Arterial Sistêmica, obesidade, vida sedentária, alimentação rica em sódio e gordura, uso moderado de álcool, uso do tabaco e o estresse. As intervenções cirúrgicas hemodinâmicas mais frequentes foram as angioplastias e angiografias.

Com a aplicação do Processo de Enfermagem na investigação comportamental, apareceu um percentual significativo de clientes que manifestavam medo em relação ao procedimento; insegurança em relação à equipe médica e clientes que não tiveram mudanças nos hábitos de vida reconhecidamente prejudiciais.

A maioria dos diagnósticos foi identificada por meio da taxonomia da NANDA I(2007-2008). Notou-se que os três diagnósticos pós-operatórios mais incidentes foram à ansiedade (100\%), seguida de risco de dor aguda $(85 \%)$ e integridade da pele prejudicada (75\%). Os demais diagnósticos tiveram percentuais abaixo de $50 \%$ entretanto demandaram cuidados específicos. Neste caso, um percentual baixo de aparecimento de um determinado diagnóstico não está diretamente relacionado a gravidade da situação de doença e não serve para determinar o critério de prioridade do cuidado. Por exemplo, no pós-operatório imediato identificar risco para diminuição da perfusão tissular renal é mais prioritário do que controlar a ansiedade do cliente.

No estabelecimento de metas na sala de recuperação pós-anestésica foram observadas as reações tardias ao contraste injetado; realizado o monitoramento do volume urinário, além do estímulo a ingestão hídrica, visando à eliminação do contraste infundido.

O plano de Intervenção caracterizou-se pelas prescrições prioritárias, de acordo com as respostas adaptativas ao cliente que dependiam da situação clínica no pósoperatório. O acompanhamento possibilitou identificar as adaptações ocorridas com cada cliente, por meio da continuidade do Processo de Enfermagem, nas visitas domiciliares. Neste sentido, a maioria dos clientes apresentou uma resposta adaptativa prejudicada, mas mantinham um comportamento de busca da saúde.

Uma dificuldade foi no entendimento quanto ao uso da teoria que norteou o trabalho, pela sua complexidade, especialmente na identificação dos estímulos manifestados pelo cliente, pois estes requerem um conhecimento clínico eficaz, ainda não desenvolvido pelas acadêmicas.

Os resultados obtidos nesta pesquisa podem contribuir para a enfermagem embasar o cuidado no Processo de Enfermagem, identificar os fatores de risco preponderantes que levam os clientes a desenvolver intercorrências cardíacas, de modo a poder atuar na prevenção destas doenças e no serviço de alta complexidade, que exige conhecimentos específicos pouco explorados durante a graduação.

\section{REFERÊNCIAS}

1. Vieira LC. Dificuldades e necessidades da equipe de enfermagem em serviços de hemodinâmica e angiografia. Arq Cienc Saude [periódico da internet]. 2009 [citado 2010 jan 26]; 16(1):21-5. Disponível em: http:// www. cienciasdasaude.famerp.br/racs_ol/vol16-1/ v16-1.htm

2. Goldman L, Ausiello D. Doenças cardiovasculares. Tratado de medicina interna. 22a ed. Rio de J aneiro: Elsevier; 2005. 


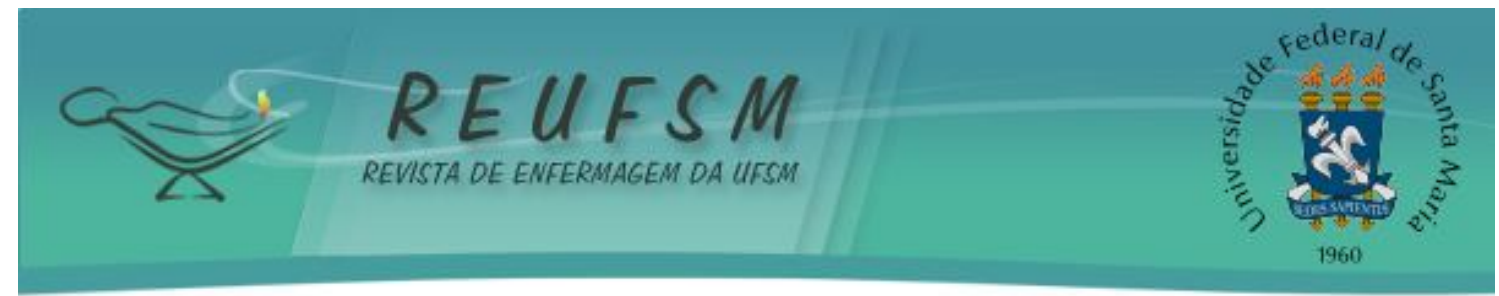

3. Luz ES, Canevaro LV, Ferreira NMPD, Campos JE. A importância do controle de qualidade em serviços de hemodinâmica e cardiologia intervencionista. Radiol Bras [periódico da internet]. 2007 [citado 2010 jan 27]; 40 (1): 27-31. Disponível em: http:/ / www. scielo.br/ pdf/ rb/ v40n1/ 06. pdf.

4. Freitas MC, Oliveira MF. Assistência de enfermagem a idosos que realizam cateterismo cardíaco: uma proposta a partir do modelo de adaptação de Calista Roy. Rev. Bras. Enferm. [periódico da internet]. 2006 [citado 2010 jan 27]; 59(5): 642-646. Disponível em: http:/ / www. scielo. br/ pdf/ reben/ v59n5/ v59n5a09. pdf.

5. Silva LP, Maurício CLP, Canevaro LV, Oliveira OS. Avaliação da exposição dos médicos à radiação em procedimentos hemodinâmicos intervencionistas. Radiol Bras [periódico da internet]. 2008 [citado 2010 jan 26]; 41(5): 319-23. Disponível em: http:// www. scielo.br/scielo.php?script=sci_arttext\&pid= S010039842008000500010\&lng=pt\&nrm=iso.

6. Leopardi MT. Teorias em enfermagem: instrumentos para a prática. Florianópolis: PapaLivro; 1999.

7. George J B et al. Teorias de enfermagem: os fundamentos à pesquisa profissional. $4^{\mathrm{a}}$. ed. Porto Alegre: Artmed; 1995.

8. Ministério da Saúde (BR). Departamento de análise da situação de saúde. Saúde Brasil. 20 anos de Sistema Único de Saúde (SUS) no Brasil [internet]. Brasília (DF) : Ministério da Saúde; 2009 [citado 2010 mai 22]. Disponível em: http:// portal.saude.gov.br/portal/arquivos/ pdf/saude_brasil_2008_web_20_11.pdf

9. Pires, DEP, Bellaguarda MLR, Zago AT, Matos E. Série Cadernos Enfermagem. Resolução n. 358, de 15 de outubro de 2009. Dispõe sobre a Sistematização da Assistência de Enfermagem em ambientes públicos ou privados em que ocorre o cuidado profissional de enfermagem e dá outras providências. Florianópolis: Quorum Comunicação; 2010. p. 89-92

10. Universidade Comunitária da Região de Chapecó. Curso de Enfermagem. Plano de ensino da disciplina de estágio curricular supervisionado II. Chapecó; 2008. 7 p.

11. Galbreath JG. Callista Roy. In: George, J B. (Org). Teorias de enfermagem: os fundamentos à pesquisa profissional. 4ª . ed. Porto Alegre: Artmed; 2000. p. 204-224.

12. Silva RP. Cuidado de enfermagem durante o processo de adaptação entre pais e recémnascidos com anomalias congênitas [dissertação]. Florianópolis : Universidade Federal de Santa Catarina, Pós Graduação em Enfermagem ; 2009. 350 p.

13. Caetano JÁ, Costa AC, Santos ZMSA, Soares E. Descrição dos fatores de risco para alterações cardiovasculares em um grupo de idosos. Texto \& Contexto Enferm [periódico da internet]. 2008 [citado 2010 jun 19]; 17(2):327-35. Disponível em: ४http:// www.scielo.br/ scielo. php?script=sci_arttext\&pid=S0104-

07072008000200015\&lng=pt\&nrm=ंso>.

14. Moreira RO, Santos RD, Martinez L, Saldanha FC, Pimenta J LAC, Feij 00 J, J ahnke N et al. Perfil lipídico de pacientes com alto risco para eventos cardiovasculares na pesquisa clínica diária. Arq Bras Endocrinol Metab [periódico da internet]. 2006 [citado 2010 jun 15]; 50(3): 481-9. Disponível em: http:// bases. bireme. br/ cgibin/ wxislind. exe/ iah/ online/ ?lsisScript=iah/ iah. xis\&src=googl e\&base=LILACS\&lang=p\&next Action $=$ nk\&exprSearch $=433742 \&$ indexSearch $=1 D$ 


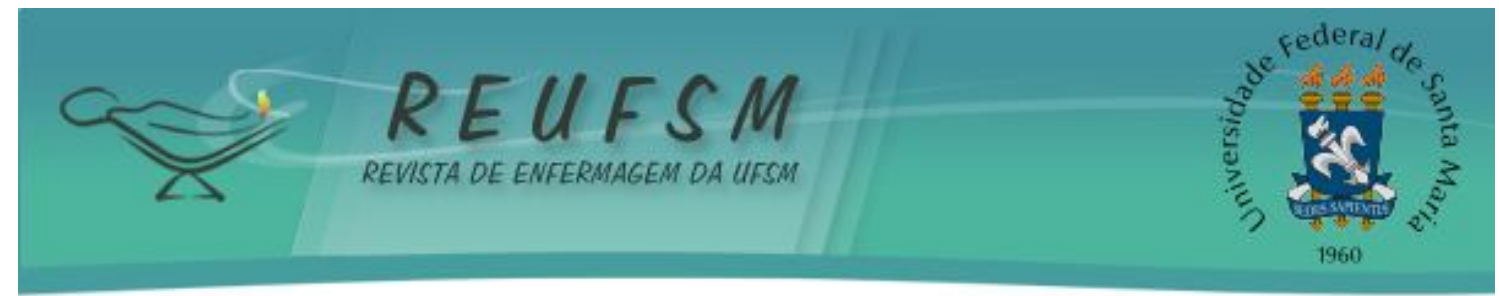

15. Lemos KF, Davis R, Moraes MA, Azzolin K. Prevalência de fatores de risco para síndrome coronariana aguda em pacientes atendidos em uma emergência. Rev Gaúcha Enferm. 2010;31(1):129-135.

16. Zanela MT, Ribeiro AB. Obesidade e Hipertensão Arterial Sistêmica. Arq Bras Cardiol [periódico da internet]. 2002 [citado jan 2011 26]; 78 (suplemento): 1-14. Disponível em: http:/ / www. scielo.br/ pdf/ abc/ v78s1/ a01v78s1. pdf

17. Smeltzer SC, Bare BG. Tratado de enfermagem médico cirúrgica. 9a ed. Rio de J aneiro: Guanabara Koogan; 2005.

18. Mesquita M, Lopes MVO, Carvalho FAF, Teixeira LFE, Barbosa IV. Teorias de enfermagem: importância da correta aplicação dos conceitos. Enferm glob [periódico da internet]. 2009 [citado 2010 jun 15]; (17):1-9. Disponivel em: বhttp:// scielo. isciii.es/ sciel o. php?script=sci_arttext\&pid=\$1695$61412009000300017 \&$ lng=es\&nrm=ंso>.

19. Michel JLM, Barros ALBL. Diagnósticos de enfermagem da NANDA: definições e classificação 2007-2008 / North American Nursing Diagnosis Association. Porto Alegre: Artmed; 2008. 396 p.

20. Lopes MVO, Araújo TL, Rodrigues DP. A relação entre os modos adaptativos de Roy e taxonomia de diagnósticos de enfermagem da NANDA. Rev Latino-am Enferm [periódico da internet]. 1999 [citado 2010 mai 15]; 7(4):97-104. Disponível em: http:/ / www. scielo.br/ scielo.php?script=sci_arttext\&pid=S0104$11691999000400013 \&$ Ing=pt\&nrm=jso\&tlng=pt.

Data de recebimento: $28 / 01 / 2011$

Data de aceite: 29/03/2011

Contato com autora responsável: Ivete Maroso Krauzer.

Endereço: Rua Marechal J osé Bormann 426 D. Edifício Vivaldi, apto 401. Chapecó-SC.

CEP 89801-050

E-mail: ivetemaroso@hotmail.com 